\title{
Detection and Transmission of EEG Signal Using VLC
}

\author{
Dhanalakshmi $\mathrm{A}^{\mathrm{a}, 1}$, Priyadarshni $\mathrm{S}^{\mathrm{b}}$, Kayalvizhi $\mathrm{P}^{\mathrm{b}}$, Devi Priya $\mathrm{G}^{\mathrm{b}}$, and Oviya $\mathrm{S}^{\mathrm{b}}$ \\ ${ }^{a}$ Associate Professor, Dept of E\&I, Panimalar Engineering College, Chennai, India \\ ${ }^{b} U G$ Scholar, Dept of E\&I, Panimalar Engineering College, Chennai, India
}

\begin{abstract}
The healthcare and the management of quality is very essential part to the recent period of medical environment. To enhance these, to make sufferer easy with timely and best remedial care.EEG systems are closely relevant on the performance using wireless system because of its more capabilities. But it has various disadvantages like line frequency interference and signal loss,. Currently there is usage of RF in healthcare environment and medical test centre which have dangerous for the health of the patients. So we are in need of alternative eco-friendly source of communication in health care. So the alternative way is proposed here is the VLC (Visible Light Communication) transmission technology that uses white LED for data transmission which has efficiency, reliability and high precision. The ADC produces data stream of EEG signal which is modulated using OOK .Here LED works as a transmitter and photodiode with sensor works as receiver and uses computer simulation for further evaluation.
\end{abstract}

Keywords. VLC, EEG, LED, Wi-Fi, LiFi, Electrodes

\section{Introduction}

VLC is a technology that deals with the idea of data transmission through signal in terms of light rays to transmit and receive messages within a short distance. The data can be transmitted through LED lighting for the replacement of radio frequencies. In addition, VLC has $\mathrm{s}$ lots of advantage over RF since VLC provides fast data transmission and high bandwidth. VLC makes use of visible light between the bandwidth range of 780 to $375 \mathrm{~nm}$ and this range is normal visible to the human eye. The data can be emitted into single units of ones and zeros that are transmitted as either low or high signals. In VLC, the data transmitted at fast turning of light on and off. Anyway, this data transmission depends upon how fast the light goes from on and off state.LED lighting is used in the configurations of VLC for transmission of data. VLC consists of a receiver (photo detector), a transmitter (LED's) and a communication channel.

From the source, an enhanced photodiode is attached to the receiver for converting light into a current and transmitted through serial port connector (RS232) which is connected to the personal computer and using MATLAB the required signal can be obtained through graph. In medical centers, several number of machines will be benefited from VLC as while interference obtained electromagnetic in terms of radio

\footnotetext{
${ }^{1}$ A.Dhanalakshmi , Associate Professor department of E\&I

Email: dhanalakshmi248@gmail.com
} 
waves from other machines. So it reduces disruption from any other devices using radio waves, hence it becomes easy to operate. VLC-unlike Wi-Fi in which radio frequency is used, VLC merely based on the light in order to function. Due to this, data is transmitted faster and wiring cost is extremely saved. Visible Light Communication (VLC) is one of the fastest technologies that can transmit data through LED. The Electroencephalography (EEG) machine s place an important role in brain examination and study. The electrode placed in human brain which can be detected through the EEG sensor the signals are transmitted to the analog to digital converters in the ARMLPC2148.Through port1 the EEG values from the converters is received by the UART2 receiver and transmitted through Iot. The EEG values from port0 are transmitted through UART1 transmitter to the VLC transmitter which is LED, through the optical communication the data can be transmitted in the form of current. On the receiver side the photodiode which detects through Line Of Sight (LOS) method from the light source, the detected signal from the photodiode will transmitted through serial connector (RS232) to the personal computer (PC).

\section{Existing system}

Concurrent EEG recording gives a way to getting high fleeting goals electrophysiological information and high goals metabolic related information of the cerebrum in the equivalent test runs. Carbon wires contains cathodes and anodes (not metallic EEG terminals with wire carbon leads) are appropriate of concurrent EEG and FMRI(Functional magnetic resonance imaging) recording, since they cause low RF (radio-recurrence) warming and low weakness antiquities than metallic type cathodes. These attributes are particularly alluringly for recording EEG in the high field of MRI scanner devices. Carbon wire leads are additionally agreeable to have during the long period sessions. Notwithstanding, carbon leads have high and more cathode electrolyte possibilities contrasted with broadly utilized $\mathrm{Ag} / \mathrm{AgCl}$ (silver/silver-chloride) anodes, which will cause moderate floats in voltage. This work presents a model framework EEG recording with carbon leads anodes and a circuit that gives the moderate floats in voltage. The framework was tried for the float in voltage, RF warming, defenselessness antiquity, and also impedance, was likewise assessed in a synchronous ERP (occasion relate potential)- FMRI explore.

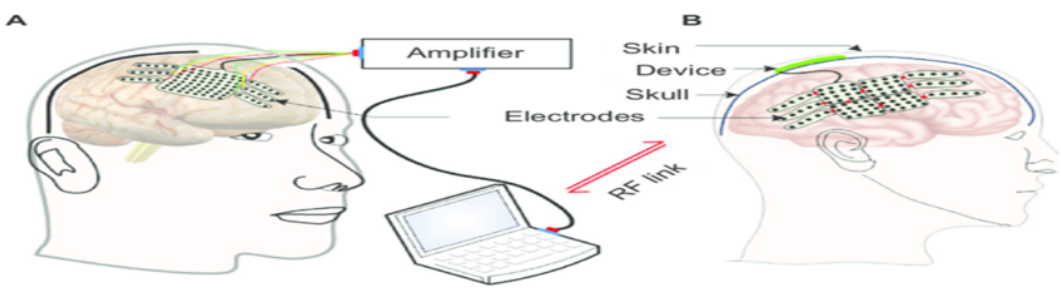

Figure 1. Representation of Wired EEG

\subsection{Wired EEG}

The utilization of carbon wire cathodes diminishes the issues. The term carbon lead cathodes made up of carbon wires and don't allude to strong EEG terminals that are appended to carbon wires. Carbon anodes cause less RF warming and less powerlessness antiques (Krakow et al., 2000) contrasted with metallic terminals. Likewise, since carbon wire anodes are exceptionally adaptable and level, subjects 
don't feel the cathodes squeezing against their heads when they lie in the scanner. Then again, carbon contains higher anode electrolyte DC possibilities than $\mathrm{Ag} / \mathrm{AgCl}$. There are regularly awkward nature among cathodes and electrolytes in and around the terminal, causing anode polarization and also conceivable immersion of the EEG speaker. This work presents an EEG recording framework which incorporates carbon wire lead cathodes and a circuit that diminishes the terminal polarization issue.

\subsection{Wireless Eeg System}

Progression of wireless technology gives more improvement in present remote electroencephalography. This technique depends on the concept obtained brainwave and its quantization limit modifies dependent on the magnitude of every EEG obtained signal. EEG, can record these activity that takes place in the brain from the brain scalp. At present the current wireless communication that has taken over previous wired solutions which is presented here in the EEG recorders. The process of monitoring the brains activities wirelessly is not only convenient but also opens up the possibility of measuring the brain activity of moving subjects. The recorded waveforms returns the activity that occurs at the cortex, the surface of the brain, and are said to be influenced by the electrical activity from the brain structures located beneath the cortex

\section{Proposed system}

Using visible light for data transmission involves huge advantages and wipes out disadvantages of transmission by means of RF waves. Scarcely any realized obvious light initiates medical issues exist today, introduction inside balance is sheltered. Since no obstruction with electromagnetic radiation happens, noticeable light can be utilized in medical clinics decisively. Moreover, noticeable light is free. No organization possesses property directly for obvious light, along these lines no sovereignty expense must be paid. Noticeable light can fill in as an altogether free foundation to base a mind boggling correspondence. VLC is generally utilized inside and transmitted light subsequently portion not leave the room when the entryways are shut and the draperies on, which makes VLC a sheltered innovation if the sender means to transmit classified information.VLC system have a lot of advantages in environments susceptible to EMI such as hospital, mines by using VLC communication we can avoid interference on RF signal ad by using the same system we can get elimination and communication through optical output. The most significant need that a light source has to meet so as to fill communication needs is the capacity to be turned on and off again in short interims, since this is the way information is later balanced. These principles out numerous ordinary light sources, for example, LED lights.

\subsection{Working Model}

Remote communication gives us more benefits to use EEG signals. However it may, experience different disadvantages, for example, signal loss, line frequency interference. EEG signal obtaining framework involves data acquisition, quantization amplification, encoding and digitizing finally wireless transmission. VLC innovation gives communication and lightning run of the mill LED based VLC frameworks are actualized utilizing a force balance and direct recognition (IM/DD) plot with a view 
(LOS) design because of its enlightenment reason. Right now, a photodiode is utilized to change over the episode optical sign force into a corresponding current. Because of the change in quick force, DD is the main conceivable sign recuperation procedure of the sign. At the point when EEG sensor which identified the sign from the mind the simple signs which is given to the ADC. The information stream is framed after the ADC of the caught EEG signal is regulated utilizing OOK. At that point the information grouping is transmitted by means of a white shading segment of the LED cluster. At the beneficiary, the photodiode is introduced, being furnished with the sensor. PC reproduction is completed to assess the viability of the proposed work plan for the transmission of EEG signals. In the transmitter, IM is executed through the modulation of the transmitting signal into the instantaneous values in optical intensity of the LED by controlling the brilliant power with the forward current through the LED. High regulation frequencies components are used to avoid flickering, which can have unfavorable health impacts. At the receiver end, the transmitted signal is reconstructed and utilizing direct detection (DD).

\subsection{EEG Signal}

An EEG can be used to help and recognize the potential issues related with this movement. EEG tracking and recording brain wave. Little level mental plates called electrodes are attached in the scalp with wire leads. The signal from the brain will be transmitted through anode and cathode.

\subsection{Analog to digital converter}

The EEG signal from the sensor will be to the ADC. LPC2148 has two inbuilt 10-bit ADC. Here the liquid crystal display (LCD) is the received end device. This successive approximation process requires a clock less than or equal to $4.5 \mathrm{MHz}$. We can just adjust clock using clock divider settings. Both ADCs in LCP2148 convert analog signals in the range of $0 \mathrm{~V}$ to $3 \mathrm{~V}$.

\section{$3.4 \mathrm{IoT}$}

The Internet of things (IoT) is the system of regular objects-physical related things fabricated with gadgets, programming, sensors, and connectivity enabling data exchange. Fundamentally, a computer is appended to the device, allowing data exchange to and from that device. May be lights, toasters, coolers, watches, fans, planes, trains, autos, or whatever else around us, PC can be joined with it for the acknowledgement of input (particularly object control) or to assemble and create educational yield (normally object status or other tactile information). This implies PCs will be penetrating all around us - omnipresent inserted registering gadgets, exceptionally recognizable, interconnected over the Internet..

\subsection{Modulation}

Create a PWM in LPC2148 development board at a frequency. Pulse width modulation is a method to obtain simple analog result with digital. Digital control mechanism is 
utilized to make a square waveform; a signal is exchanged among on and off. This onoff example can simulate voltages in the middle of 0 to $5 \mathrm{~V}$. The Interfacing PWM with LPC2148 program is extremely basic, which produces a heartbeat design in a specific recurrence. An ADC signal is accustomed to fluctuating the obligation pattern of PWM signal.

\subsection{LIFI Transmitter}

The ARM controller will at that point adjust the information utilizing digital modulation like pulse width modulation (PWM) technique for logic 1s and 0s. The transmitter section comprises of a sending end side gadget, a power is supplied to a controller by power supply, and simple hardware connected the driver and LED light.



Figure 2. Lifi Receiver Functional Block Diagram

\section{Results and discussion}

To confirm a dependable and exact transmission of the EEG biomedical data, we examined the EEG signal transmission of EEG signal for the test arrangement. The output of the proposed framework system that utilized a white color LED and combined selection along with the color filters. Obviously the transmitted information is received exactly with more reliability.

As part of additional assessment of the effectiveness of our proposed framework, the kept on performing simulations under digital communication environment, standard additive white Gaussian noise (AWGN) and figured out the biterror rate (BER) at various levels of signal to-noise ratio (SNR).

\section{Conclusions and Further Scope}

The data transmission of EEG signal using advanced VLC is proposed. In many health care environments, even cell phones are not permitted due to concern radio frequency interference with sensitive electronic equipment or devices. Visible light communication is an ideal method to avoid such interference and provide less expensive, more reliable, communication between equipment or patience and monitoring stations. LIFI refers to $5 \mathrm{G}$ obvious light communication framework 
utilizing light emitting diodes as a medium for rapid communication likewise as WIFI.

At now a days where web has become a significant interest, individuals are looking for WIFI hotspots for their exercises. LIFI is a superior option in wireless communication for as it is by directional, rapid and completely arranged remote communication like WIFI. It is a subset of VLC and can be a commendation to RF communication. This report proposes a survey on LIFI technology. LIFI has much more capacity in terms of band with invisible region hence it does not interfere with other communication which uses radio frequency range, without taking its frequency bands. It has more times superior speed than WIFI technology. This proposed technology has brought not only greener but more safety for medical environment and cheaper for future wireless communication.

\section{References}

[1] H. Hong, Y. Ren, and C. Wang .Information illuminating system for healthcare institution . in Proc. 2nd Int. Conf. Bioinformat. Biomed. Eng., May 2008, p. 801-804.

[2] P. P. Han, A. Sewaiwar, S. V. Tiwari, and Y.-H. Chung.Color clustered multiple-input multiple-output visible light communication. J. Opt. Soc. Korea, vol. 19, no. 1, p. 74-79, 2015.

[3] N. Lawrentschuk and D. M. Bolton.Mobile phone interference with medical equipment and its clinical relevance: A systematic review. Med. J. Aust., vol. 181, no. 3, pp. 145-149, 2004.

[4] M. H. Nezhad, K. S. Subari, and M. Yahyavi .Improvement of wireless transmission system performance for EEG signals based on development of scalar quantization. J. Elect. Bioimpedance, vol. 4, pp. 62-72, Dec. 2013

[5] E. Pisek, S. Rajagopal, and S. Abu-Surra .Gigabit rate mobile connectivity through visible light communication . in Proc. IEEE Int. Conf. Commun., Jun. 2012, pp. 3122-3127.

[6] S. Rajagopal, R. D. Roberts, and S.-K. Lim .IEEE 802.15.7 visible light communication: Modulation schemes and dimming support .IEEE Commun. Mag., vol. 50, no. 3, pp. 72-82, Mar. 2012.

[7] Y. Tan, S.-J. Jung, and W.-Y. Chung .Real time biomedical signal transmission of mixed ECG signal and patient information using visible light communication. in Proc. 35th Annu. Int. Conf. IEEE EMBC, Jul. 2013, pp. 4791-4794.

[8] Etoolbox. Bioelectromagnetism MATLAB Toolbox. [Online]. Available: http://eeg.sourceforge.net, accessed Feb. 10, 2015.

[9] A Hybrid petri Net Approach for Polyethylene Terepthalatae bottle manufacturing system B.Muthuraj,V.Mahesh, R.Senthil Journal of computational and Thearetical Nanoscience-2016.

[10] Simulation Of Three Tank System Using Hybrid Perti Net For Batch Process Dr. R.Senthil B.Muthuraj, Dr.V.Mahesh 2015/11/2 International Journal of Applied Engineering Research 4789-4798 Research India 